\title{
Glutaminolysis dynamics during astrocytoma progression correlates with tumor aggressiveness
}

Yollanda E. Moreira Franco ${ }^{1 *}$ (D), Maria Jose Alves ${ }^{1 \dagger}$, Miyuki Uno ${ }^{1}$, Isabele Fattori Moretti ${ }^{1}$, Marina Trombetta-Lima ${ }^{1,2}$, Suzana de Siqueira Santos ${ }^{3}$, Ancely Ferreira dos Santos ${ }^{4}$, Gabriel Santos Arini ${ }^{4}$, Mauricio S. Baptista ${ }^{4}$, Antonio Marcondes Lerario ${ }^{5}$, Sueli Mieko Oba-Shinjo ${ }^{1}$ and Suely Kazue Nagahashi Marie ${ }^{1}$

\begin{abstract}
Background: Glioblastoma is the most frequent and high-grade adult malignant central nervous system tumor. The prognosis is still poor despite the use of combined therapy involving maximal surgical resection, radiotherapy, and chemotherapy. Metabolic reprogramming currently is recognized as one of the hallmarks of cancer. Glutamine metabolism through glutaminolysis has been associated with tumor cell maintenance and survival, and with antioxidative stress through glutathione (GSH) synthesis.
\end{abstract}

Methods: In the present study, we analyzed the glutaminolysis-related gene expression levels in our cohort of 153 astrocytomas of different malignant grades and 22 non-neoplastic brain samples through qRT-PCR. Additionally, we investigated the protein expression profile of the key regulator of glutaminolysis (GLS), glutamate dehydrogenase (GLUD1), and glutamate pyruvate transaminase (GPT2) in these samples. We also investigated the glutathione synthase (GS) protein profile and the GSH levels in different grades of astrocytomas. The differential gene expressions were validated in silico on the TCGA database.

Results: We found an increase of glutaminase isoform 2 gene (GLSiso2) expression in all grades of astrocytoma compared to non-neoplastic brain tissue, with a gradual expression increment in parallel to malignancy. Genes coding for GLUD1 and GPT2 expression levels varied according to the grade of malignancy, being downregulated in glioblastoma, and upregulated in lower grades of astrocytoma (AGII-AGIII). Significant low GLUD1 and GPT2 protein levels were observed in the mesenchymal subtype of GBM.

(Continued on next page)

\footnotetext{
* Correspondence: yollanda.moreiraf@usp.br

†Yollanda E. Moreira Franco and Maria Jose Alves contributed equally to this work.

'Laboratory of Molecular and Cellular Biology (LIM 15), Department of Neurology, Faculdade de Medicina FMUSP, Universidade de São Paulo, São Paulo 01246-903, Brazil

Full list of author information is available at the end of the article
}

(C) The Author(s). 2021 Open Access This article is licensed under a Creative Commons Attribution 4.0 International License, which permits use, sharing, adaptation, distribution and reproduction in any medium or format, as long as you give appropriate credit to the original author(s) and the source, provide a link to the Creative Commons licence, and indicate if changes were made. The images or other third party material in this article are included in the article's Creative Commons licence, unless indicated otherwise in a credit line to the material. If material is not included in the article's Creative Commons licence and your intended use is not permitted by statutory regulation or exceeds the permitted use, you will need to obtain permission directly from the copyright holder. To view a copy of this licence, visit http://creativecommons.org/licenses/by/4.0/ The Creative Commons Public Domain Dedication waiver (http://creativecommons.org/publicdomain/zero/1.0/) applies to the data made available in this article, unless otherwise stated in a credit line to the data. 


\begin{abstract}
(Continued from previous page)
Conclusions: In glioblastoma, particularly in the mesenchymal subtype, the downregulation of both genes and proteins (GLUD1 and GPT2) increases the source of glutamate for GSH synthesis and enhances tumor cell fitness due to increased antioxidative capacity. In contrast, in lower-grade astrocytoma, mainly in those harboring the IDHI mutation, the gene expression profile indicates that tumor cells might be sensitized to oxidative stress due to reduced GSH synthesis. The measurement of GLUD1 and GPT2 metabolic substrates, ammonia, and alanine, by noninvasive MR spectroscopy, may potentially allow the identification of IDH ${ }^{\text {mut }} \mathrm{AGIl}$ and AGIII progression towards secondary GBM.
\end{abstract}

Keywords: Glutaminolysis, GBM, Low-grade astrocytoma, IDH1 mutation, Astrocytoma progression

\section{Background}

Cancer is among the leading causes of death worldwide [1]. Although the tumors of the central nervous system (CNS) are less frequent, representing about $3 \%$ of all tumors, they are among the most aggressive [2, 3]. Gliomas, which originate from glial cells or their precursors, represent more than $80 \%$ of primary brain tumors [4-6]. Glioblastoma (GBM), the most frequent adult malignant glioma and classified as a WHO grade IV astrocytoma, has been stratified according to the molecular profile as proneural, classical, and mesenchymal subtypes $[7,8]$, which partially predict the clinical outcome. The proneural subtype characterized by the presence of $I D H$ mutation has been associated with a better prognosis [9], while the mesenchymal subtype with NF1 or RB1 mutations has presented the worst outcome, with an average overall survival of 8-11 months [10]. More recently, an impact of the mutational landscape on the response to immunotherapy and on the acquired resistance to temozolomide (TMZ) has been demonstrated in gliomas [11].

The capacity of cancer cells to reprogram their metabolisms to support rapid proliferation is another cancer hallmark with prognostic impact [12, 13]. Interestingly, metabolic enzymes with high catalytic activity are found upregulated in different kinds of tumor and are associated with poor survival [14]. Glutamine (Gln) metabolism is upregulated by various oncogenic signaling pathways [15] and is relevant in cancer development due to its involvement in mTOR signaling, autophagy, and antioxidative stress and as a source of glutathione (GSH) and for anaplerosis [12, 13, 16]. Moreover, Gln uptake and the rate of glutaminolysis are known to be related to tumor growth [17-19]. Besides, we previously have observed that Gln transporters are upregulated in astrocytoma [20]. The dependence of cancer cells on Gln makes glutaminolysis an attractive cancer therapy target $[15,21-23]$. Gln is a non-essential amino acid, consumed largely by proliferating cancer cells in vitro, which are often dependent on extracellular Gln for survival [24]. Gln carbon contributes to aspartate, glutamate (Glu), and tricarboxylic acid (TCA) cycle metabolites via glutaminolysis [15]. High rates of glutaminolysis support rapid proliferation by supplying precursors to low-flux biosynthetic pathways [24]. Current attempts to target glutaminolysis clinically have focused largely on inhibiting glutaminase. Chemical inhibitors have been found to decrease cancer cell proliferation in both in vitro/in vivo models [25-27].

The metabolic ending of glutamine-derived Glu is, apart from $\alpha$-ketoglutarate ( $\alpha-K G)$, lactate and GSH being an important nitrogen donor for cell growth and proliferation [28, 29]. Additionally, studies about IDH mutation showed significantly reduced levels of Gln and Glu levels were, which implies replenishment of $\alpha-K G$ by glutaminolysis. Consequently, wild-type (wt) gliomas presented high levels of intracellular Glu, which is released via the $\mathrm{Gln} /$ cysteine antiporter System $\mathrm{X}_{\mathrm{C}}{ }^{-}$in exchange for cysteine. GSH is considered a potent antioxidant and the main factor responsible for treatment resistance in gliomas or other neoplastic cells [30, 31]. Therapeutic attempts have been aimed at GSH depletion by inhibiting the $\mathrm{X}_{\mathrm{C}}{ }^{-}$transporter [32], which is responsible for counter-transport of Glu and cysteinea substrate-limiting GSH synthesis [30, 31]. This exchange is favorable for the cancer cells because Cys is a major component of the antioxidant GSH, which in turn is an antagonist of reactive oxygen species (ROS) [28].

In the present study, we analyzed the expression profile of the genes related to glutaminolysis in different grades of astrocytomas and, more specifically, in the molecular subtypes of GBM, and lower malignant grades of astrocytoma regarding IDH1 mutation status. We searched for differential features of glutaminolysis related to GBM aggressiveness and malignant progression of low-grade astrocytomas with $I D H 1$ mutation, which may help to better characterize the metabolic features associated with GBM aggressiveness and to tumor malignant progression.

\section{Methods}

Tissue sample and ethical statement

Samples were snap-frozen in liquid nitrogen immediately following surgical removal and macro dissected before RNA extraction. A 4- $\mu$ m-thick cryosection of each sample was stained with hematoxylin-eosin and analyzed 
under a light microscope for assessment of necrotic, cellular debris, and NN areas (in tumoral samples). For gene expression, we analyzed 153 human astrocytoma samples stratified according to the WHO classification (2007) [33] as 23 astrocytomas grade I (AGI), 26 astrocytomas grade II (AGII), 18 astrocytomas grade III (AGII I), and 86 GBM. Non-neoplastic brain samples (NN) were used as control (22 cases). For the GLS protein analysis, NN (5), AGI (4), AGII (4), AGIII (2), and GBM (6) were evaluated. For GLUD1 and GPT2 protein analysis, we explored AGII-IDH $H^{\mathrm{wt}}$ (4), AGII-IDH $H^{\mathrm{mut}}$ (6), GBM of mesenchymal subtype (GBM-MS) (7), and GBM of proneural subtype (GBM-PN) (5) samples. For glutathione synthetase (GS) protein analysis, we explored AGII-IDH $H^{\mathrm{wt}}(4)$, AGII-IDH ${ }^{\mathrm{mut}}$ (4), GBM-MS (4), and GBM-PN (4). Tumor samples were obtained from surgery of patients treated by the Neurosurgery Group of Department of Neurology at Hospital das Clinicas at the School of Medicine of University of São Paulo, from 2000 to 2007. NN brain tissue samples were collected from epilepsy patients subjected to temporal lobectomy.

\section{Cell culture}

The U87MG cell line was acquired from ATCC and authenticated by short tandem repeats (STR) analysis using GenePrint 10 System (Promega, Madison, WI). Cells were cultured in monolayer with DMEM medium (Dulbecco's modified Eagle's medium, Thermo Fisher Scientific, Carlsbad, CA), 10\% fetal bovine serum, and $100 \mu \mathrm{g} /$ $\mathrm{ml}$ streptomycin and $100 \mathrm{IU} / \mathrm{ml}$ penicillin.

\section{RNA extraction and cDNA synthesis}

Total RNA was extracted from frozen tissues (tumor and $\mathrm{NN}$ ) using the RNeasy Mini Kit (Qiagen, Hilden, Germany) following the manufacturer's instructions. The RNA concentration and purity were evaluated by NanoDrop, and ratios of 260/280 measures ranging from 1.8 to 2.0 were considered satisfactory for purity standards. RNA quality was checked by electrophoresis in agarose gel. A conventional reverse transcription reaction was performed to yield single-stranded cDNA. The first strand of cDNA was synthesized from $1 \mu \mathrm{g}$ of total RNA previously treated with 1 unit of DNase I (FPLC-pure, GE Healthcare, Uppsala, Sweden) using random and oligo (dT) primers, RNase inhibitor, and SuperScript III reverse transcriptase according to the manufacturer's recommendations (Thermo Fisher Scientific). The resulting cDNA was subsequently treated with 1 unit of RNase $\mathrm{H}$ (GE Healthcare), diluted with TE buffer, and stored at $-20{ }^{\circ} \mathrm{C}$ until later use.

\section{Analysis of gene expression by quantitative real-time PCR (qRT-PCR)}

The relative expression levels of genes involved in the glutaminolysis pathway GLS, GLSiso1, GLSiso2, GLS2,
GLUD1, GOT1, GOT2, and GPT2 were analyzed by qRT-PCR, using the SYBR Green approach. The expression of ASCT2 and LAT1 genes were previously described by our group [20]. A geometric mean of three suitable reference genes was used for normalizing the quantitative data: hypoxanthine phosphoribosyltransferase (HPRT), glucuronidase beta (GUS $\beta)$, and TATA box binding protein $(T B P)$ [34]. The primers were designed to amplify $80-120$ bp amplicons, with a melting temperature of $60{ }^{\circ} \mathrm{C}$ and were synthesized by IDT (Integrated DNA Technologies, Coralville, IA). The primers information is described in Table 1.

To ensure the efficiency of amplification and analysis of melting curves, which gave a single peak for all PCR products, standard curves with varying concentrations of the primer pairs of each gene were performed. The optimal primer concentration was determined as the lower concentration which did not affect the cycle threshold (Ct) and displayed the maximum amplification efficiency while minimizing non-specific amplification. Additionally, the amplified PCR product sizes were checked by agarose gel electrophoresis. The SYBR Green I amplification mixtures $(12 \mu \mathrm{l})$ were composed of cDNA, Power SYBR Green I Master Mix (Thermo Fisher Scientific), and the reverse and forward primers. The qRT-PCR was done in duplicate using the ABI Prism 7500 (Thermo Fisher Scientific) as follows: $2 \mathrm{~min}$ at $50{ }^{\circ} \mathrm{C}, 10$ min of polymerase activation at $95{ }^{\circ} \mathrm{C}$, and 40 cycles of $15 \mathrm{~s}$ at $95{ }^{\circ} \mathrm{C}$ and $1 \mathrm{~min}$ at $60{ }^{\circ} \mathrm{C}$. The following equation was applied to calculate gene expression in tumor and $\mathrm{NN}$ tissue samples: $2^{-\Delta \mathrm{Ct}}$, where $\Delta \mathrm{Ct}=\mathrm{Ct}$ of a specific gene-geometric mean $\mathrm{Ct}$ of housekeeping genes [35].

\section{Analysis of protein expression by western blotting}

The samples and U87MG cells were homogenized with RIPA lysis buffer (50 mM Tris-HCl, 1\% NP-40, 0.25\% $\mathrm{Na}$ deoxycholate, $150 \mathrm{mM} \mathrm{NaCl}, 1 \mathrm{mM}$ EDTA) supplemented with a cocktail of protease inhibitors (Sigma-Aldrich, St Louis, MO). The protein concentration was determined using the Bradford reagent. All samples $(20 \mu \mathrm{g}$ protein) were resolved by electrophoresis on 4-12\% gradient gels in SDS-PAGE using electrophorese buffer NuPAGE MOPS SDS (Thermo Fisher Scientific) and transferred onto PVDF membrane by iBlot Dry Blotting System (Thermo Fisher Scientific). Then the remaining binding sites of the membranes were blocked with skimmed milk powder solution at $5 \%$ diluted in Tris-buffered saline and $0.1 \%$ Tween 20 (TBST). Subsequently, the membranes were incubated overnight with the primary antibody, anti-GLS (1:1,000), anti-GS (1:2,000) from Abcam (Cambridge, MA), and anti-GLUD1, anti-GPT2 (1:1, 000) from Thermo Fisher and then diluted in TBST with $5 \%$ bovine serum albumin (BSA) solution. $\beta-$ 
Table 1 The primer sequences and the concentration used in qRT-PCR

\begin{tabular}{|c|c|c|c|}
\hline Genes & Forward primer $\left(5^{\prime}-3^{\prime}\right)$ & Reverse Primer $\left(5^{\prime}-3^{\prime}\right)$ & Concentration (nM) \\
\hline GLS & CAGGGCAGTTTGCTTTCCAT & GAGACCAGCACATCATACCCAT & 200 \\
\hline GLSiso 1 & GCAGAGGGTCATGTTGAAGTTGT & GGTGTCCAAAGTGCAGTGCTT & 200 \\
\hline GLSiso2 & ATCCTCGAAGAGAAGGTGGTGA & GCAAGTTCTTGTTGGAGACTITCA & 400 \\
\hline GLS2 & ATCCTCGAAGAGAAGGTGGTGA & ATGGCTGACAAGGCAAACCT & 200 \\
\hline GLUD1 & TGGCATACACAATGGAGCGT & TCTCAATGGCATTAACATAGGCA & 400 \\
\hline GOT1 & CTGTGCCCAGTCCTTCTCCA & GATGCTCTCAGGTTCTTTTCCAA & 400 \\
\hline GOT2 & CTTGAGGTTGGAGACCAGTTGAGT & GATTGCTGCTGCCATTCTGA & 400 \\
\hline GPT2 & GGCTITGGGCAGAGGGAA & TCACGCGTACTTCTCCAGGAA & 200 \\
\hline HPRT & TGAGGATTTGGAAAGGGTGT & GAGCACACAGAGGGCTACAA & 200 \\
\hline GUS $\beta$ & AAATACGTGGTTGGAGAGCTCATT & CCGAGTGAAGATCCCCTTITTA & 400 \\
\hline TBP & AGGATAAGAGAGCCACGAACCA & CTTGCTGCCAGTCTGGACTGT & 200 \\
\hline
\end{tabular}

actin (1:5.000) (Sigma-Aldrich) was used as a loading control. The membranes were incubated with peroxidase-conjugated secondary antibody anti-rabbit and anti-mouse (1:5.000) (Sigma-Aldrich), also diluted in TBST 5\% BSA. The protein levels were detected using the chemiluminescence detection method (Western Lightning Plus-ECL, Enhanced Chemiluminescence Substrate, Perkin Elmer, Waltham, MA). The detection of the chemiluminescent signal was performed in the Photo QuantLAS 4000 mini (GE Healthcare) photo documentation system and the bands were analyzed and quantified using ImageJ software (obtained from imagej.nih.gov/ij/download/).

\section{GSH measurement}

Tissue samples were resuspended in PSB-0.5\% NP40 (pH 6) and homogenized in syringes with an insulin needle 10 times. An aliquot of each sample was separated for protein quantification. Eighty microliters were processed with $250 \mu \mathrm{L}$ of cold GSH extraction buffer $\left(\mathrm{KClO}_{4}\right.$ $50 \mathrm{mM}$; EDTA $\left.10 \mathrm{mM} ; \mathrm{H}_{3} \mathrm{PO}_{4} 0.1 \%(\mathrm{v} / \mathrm{v}), \mathrm{pH} 5\right)$ and 40 $\mu \mathrm{L}$ of cold metaphosphoric acid $5 \%(\mathrm{v} / \mathrm{v})$. The samples were vortexed for $1 \mathrm{~min}$ and centrifuged at $8000 \times g(10$ $\left.\min , 4^{\circ} \mathrm{C}\right)$. The supernatants were used as 1:10 dilutions. GSH was measured using a fluorometric detection assay kit (ab138881, Abcam) assay according to the manufacturer's instructions. This assay is based on the fluorescent properties of thiol green, which is a non-fluorescent dye that becomes strongly fluorescent upon reacting directly with GSH. The fluorescence intensity was evaluated at an excitation wavelength of $490 \mathrm{~nm}$ and an emission wavelength of $520 \mathrm{~nm}$ using a 96-well plate in a spectrofluorometer (SpectraMAX M2, Molecular Devices, Sunnyvale, CA). GSH concentration was calculated by interpolation of a standard curve and results were expressed as $\mathrm{pmol} / \mu \mathrm{g}$ of total protein.

\section{TCGA data analysis}

The gene expression from the RNAseq GBM dataset was downloaded (Genomics Data Commons Data Portal-https://portal.gdc.cancer.gov/) and normalized by DEseq R software. Normalized read counts were converted to a $z$-score for heat map visualization.

\section{Statistical analysis}

Statistical analysis was conducted with SPSS for Windows, version 20.0 (IBM Corporation, Armonk, NY), and GraphPad Prism (version 5.02, San Diego, CA). Comparisons were considered statistically significant when $p<0.05$. The non-parametric Kruskal-Wallis and post hoc Dunn tests were used to analyze the differences in mRNA relative expression in different grades of astrocytomas. The correlation analysis between gene expression values was assessed by the non-parametric Spearman-rho correlation test. The variation of specificity and sensibility of gene expression levels was analyzed using the receiver operating characteristic (ROC) curve. Among the continuous variables categorized through the ROC curve, the value with the best sensitivity and specificity was chosen as the cut-off value. The area under the ROC curve (AUC) was used to measure how the expression levels could distinguish between two groups. The gene expressions were classified as hyper or hypoexpressed based on this cut-off value. The comparison of protein expression analysis was carried by two-way ANOVA and Bonferroni post-test.

\section{Results}

GAC (GLSiso2) expression increases in parallel to astrocytoma malignancy

Once Gln is transported into cells by ASCT2 (SLC1A5) and LAT1 (SLC7A5), it is converted to Glu by glutaminases (GLS and GLS2). This is a critical step, as Glu does not efficiently cross the blood-brain barrier, and brain 
interstitial Glu concentration is maintained essentially through its synthesis [36-38]. GLS presents two isoforms: KGA (GLSiso1, cytosolic) and GAC (GLSiso2, mitochondrial). The GLSiso2 is derived from an alternative exon splicing at the 3 '-end terminal, excluding the ankyrin repeats at the $\mathrm{C}$-terminus coded by the last four exons of the GLSiso1 transcript. Thus, the GLSiso2 is shorter than the GLSiso1, with a distinct C-terminal [39]. Expression analysis of transcript coding for the key enzymes involved in glutaminolysis, GLSiso1, GLSiso2, GLS2, GLUD1, GOT1, GOT2, and GPT2 was performed in our series of astrocytomas of different malignant grades and $\mathrm{NN}$ brain samples. Interestingly, although no significant differential expression of the total GLS transcripts was observed among different grades of astrocytoma compared to $\mathrm{NN}$, a significant GLSiso2 (GAC) hyperexpression was observed in all grades of astrocytoma when compared to $\mathrm{NN}(p<0.0001$ Kruskal-Wallis test, and $p<0.001$ Dunn test), with the highest expression levels detected in a set of GBM samples (Fig. 1a). Of note, GLSiso2 expression increased in parallel to the grade of malignancy $(p<0.0001$ AGII vs. AGIII, $p<$ 0.05 AGII vs. GBM, $p<0.05$ AGIII vs. GBM; Dunn test) which reflected an increase of its correlation with the gene expression levels of the glutaminolysis pathway from NN to GBM (Fig. 1a). When gene expression level correlations were analyzed, GLSiso2 expression correlated weakly only with GPT2 expression in NN, while no correlation was detected in AGI. GLSiso2 correlated negatively with GLS2, GOT1, and GOT2 in AGII and positively with GLS2 and GLSiso1 in AGIII, whereas GLSiso 2 correlated positively with all genes of the glutaminolysis pathway in GBM (Fig. 1b). All statistically significant values are demonstrated in Supplemental Table 1. Additionally, as shown in Fig. 2, the GLSiso2 expression levels presented high discriminatory power to distinguish between GBM and NN samples by ROC curve analysis (AUC $=0.919 ; 95 \% \mathrm{CI}, 0.867-0.971$ ) and between GBM and AGII, although with lower discriminatory power (AUC $=0.675 ;$ 95\% CI, 0.569-0.781).

In contrast, GLSiso1 $m R N A$ expression was significantly lower in AGI, AGII, and GBM compared to NN ( $p<0.0001$ Kruskal-Wallis test, and $p<0.05$ Dunn's test) (Fig. 1a), with discriminatory power to distinguish between GBM and NN (AUC = 0.796; 95\% CI, 0.10.308). No significant difference in its expression was detected in a pairwise comparison among different grades of astrocytoma. However, we observed a strong positive correlation between GLSiso1 and GLS2 in AGII and with GLSiso2 in AGIII, as well as with GLSiso2, GLUD1, GOT1, GOT2, and GPT2 in GBM cases. Similarly, GLS2 hypoexpression was observed in astrocytoma of all malignant grades compared to $\mathrm{NN}(p<0.0001$ KruskalWallis test, and $p<0.00001$ for all astrocytoma grades and NN Dunn test), and its expression level presented the power to distinguish between GBM and NN (AUC = 0.791; 95\% CI, 0.000-0.06) and to distinguish between GBM and AGII, but with lower discriminatory power (AUC $=0.65$; 95\% CI, 0.221-0.48).

GLS isoforms expression was also investigated at the protein level, and we confirmed a differential expression of GLSiso1 and GLSiso2 in NN and astrocytomas of different malignant grades (Fig. 3). Whereas GLSiso1 was present in all NN and diffusely infiltrative astrocytoma (grade II to IV) samples, with higher abundance in NN samples in comparison to GBM samples $(p<0.001$ ANOVA two-way, with Bonferroni post-test). In contrast, GLSiso2 was mostly detected in GBM cases and only slightly detected in NN samples. GLS isoforms were detected in the U87MG cell line-a GBM mesenchymal subtype cell line (Fig. 3).

\section{GPT2 downregulation in the GBM mesenchymal subtype correlated to upregulation of genes involved in GSH synthesis}

Once Glu is synthesized it can be converted to $\alpha-K G$, an intermediate of the TCA cycle, by glutamate dehydrogenase (GLUD1) and glutamate transaminases, as glutamate oxaloacetate transaminases (GOT1-cytosolic and GOT2-mitochondrial) and glutamate pyruvate transaminases (GPT1-cytosolic and GPT2-mitochondrial), which transfer amino groups from oxaloacetate or from pyruvate to generate $\alpha-K G$ and aspartate or alanine, respectively [17]. The expression levels of GLUD1, GOT1, GOT2, and GPT2 were differentially expressed in astrocytomas compared to NN $(p<0.0001$ KruskalWallis test for GLUD1, GOT1, and GPT2 and $p<0.002$ for GOT2) (Fig. 1a). Interestingly, GLUD1 expression was significantly decreased in GBM compared to AGII $(p<0.005$ Dunn test), and its expression level presented discriminatory power to distinguish between GBM and $\mathrm{NN}$ (ROC AUC $=0.770 ; 95 \% \mathrm{CI}, 0.128-0.332$ ) and between GBM and AGII (ROC AUC $=0.742 ; 95 \% \mathrm{CI}$, $0.147-0.368$ ) (Fig. 2).

In contrast, GOT2 expressions increased according to malignancy ( $p<0.001$ AGII vs. AGIII and $p<0.01$ AGII vs. GBM, Dunn test) (Fig. 1a) and presented discriminatory power to distinguish between GBM and AGII $($ AUC $=0.709 ; 95 \%$ CI, 0.608-0.810) (Fig. 2). GPT2 expression also increased significantly according to malignancy (AGI relative to AGII $p<0.001$, to AGIII $p<$ 0.0001 , to GBM $p<0.05$; AGII vs AGIII $p<0.05$, AGIII vs GBM $p<0.005$; Dunn test), besides, the only different expression level in comparison with NN was AGIII $(p<$ 0.0001) (Fig. 1a). Although GOT1 expression levels differed significantly between $\mathrm{NN}$ and astrocytoma, with discriminatory power to distinguish GBM from $\mathrm{NN}$ (ROC AUC $=0.902 ; 95 \%$ CI, 0.038-0.159), no significant 


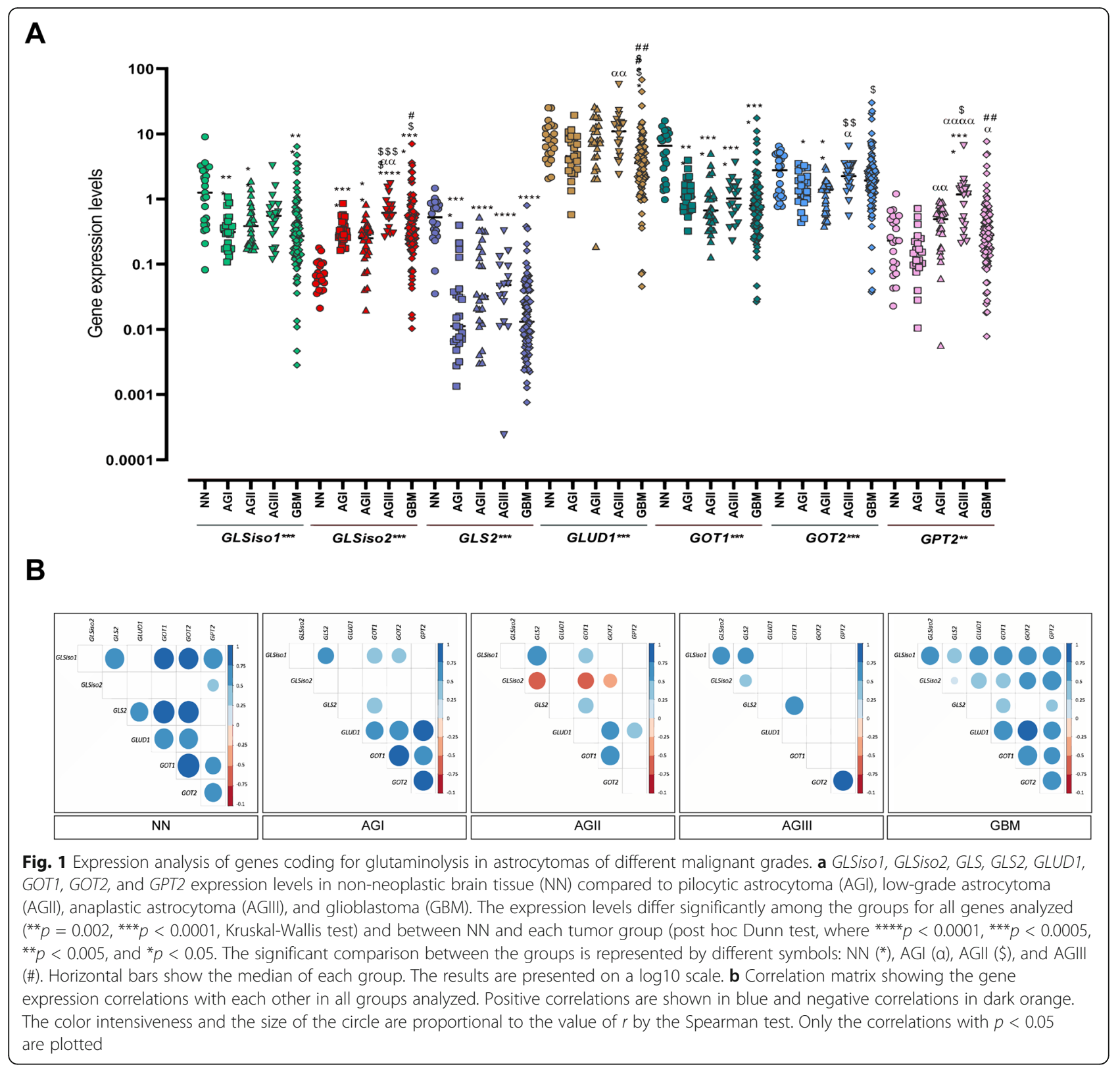

difference of GOT1 expression levels was detected among the astrocytoma grades of malignancy (Fig. 1a).

Considering the natural history of malignancy progression of malignancy from AGII to GBM, an upregulation of GLSiso2, GOT2, and GPT2 expression levels were observed, in contrast to the downregulation of GLUD1. However, a large spreading of their expressions was detected in GBM, consistent with the well-known heterogeneity observed in GBM. Therefore, we analyzed the expression levels in GBM cases classified according to the molecular subtypes in proneural (PN), classical (CS), and mesenchymal (MS) subtypes [40]. Our cohort comprised $14 \mathrm{PN}, 38 \mathrm{CS}$, and $14 \mathrm{MS}$ cases. We found a statistical difference for GLS2 expression among the groups
( $p<0.005$, Kruskal-Wallis test) and comparing two groups: PN vs MS ( $p<0.05$, Dunn test) and CS vs MS ( $p<0.05$, Dunn test), with lower expression detected in the MS subtype (Supplemental Figure 1).

To validate these findings, we analyzed gene expression in silico in a larger database. The TCGA GBM database with gene expression from RNAseq comprising 37 PN (8 G-CIMP and 29 non-G-CIMP), 38 CS, and $48 \mathrm{MS}$ cases. GPT2 expression levels varied significantly among the GBM subtypes ( $p<0.0001$, Kruskal-Wallis test) with lower levels in MS than G-CIMP $(p<0.0005)$, PN $(p<0.05)$, and CS $(p<0.05$, Dunn test). GLUD1 expression levels also varied significantly amongst GBM subtypes ( $p=0.05$, Kruskal-Wallis test) with significant 


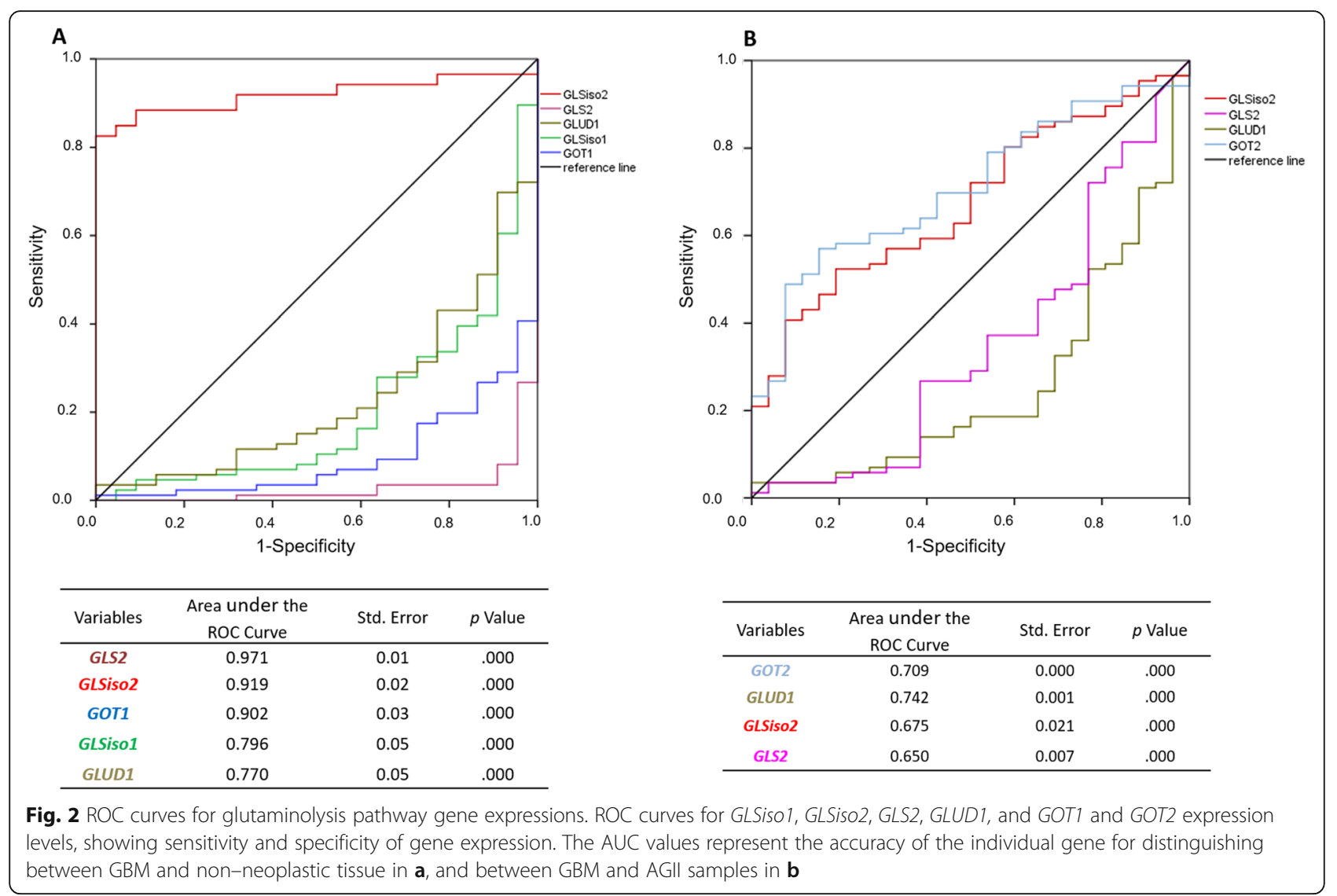

higher levels in G-CIMP compared to PN $(p<0.01)$ and to MS ( $p<0.005$, Dunn test). Although no statistical significance was observed of GLS expression among GBM subtypes, a trend of increase of its expression was noted in the MS subtype (Fig. 4a). The expression levels of $G L S$ isoforms were not available in this dataset. The TCGA data analysis showed the downregulation of GLUD1 and GPT2 involved in the conversion of Glu to $\alpha-K G$. GLUD1 differed statistically in G-CIMP to MS $(p<0.01)$. Particularly, GPT2 differed when comparing all groups with the MS subtype of GBM (G-CIMP-MS $p<0.001$; PN-MS $p<0.01$; CS-MS $p<0.05$ ).

The downregulation of both genes GLUD1 and GPT2 suggest that the intracellular availability of Glu is increased, especially in the MS subtype of GBM, which led us to investigate another important Glu metabolism pathway: GHS. To this purpose, we selected the genes related to GSH pathway, glutamate-cysteine ligase modifier subunit (CGLM), gamma-glutamylcyclotransferase (GGCT), glutathione S-transferase mu 4 (GSTM4),

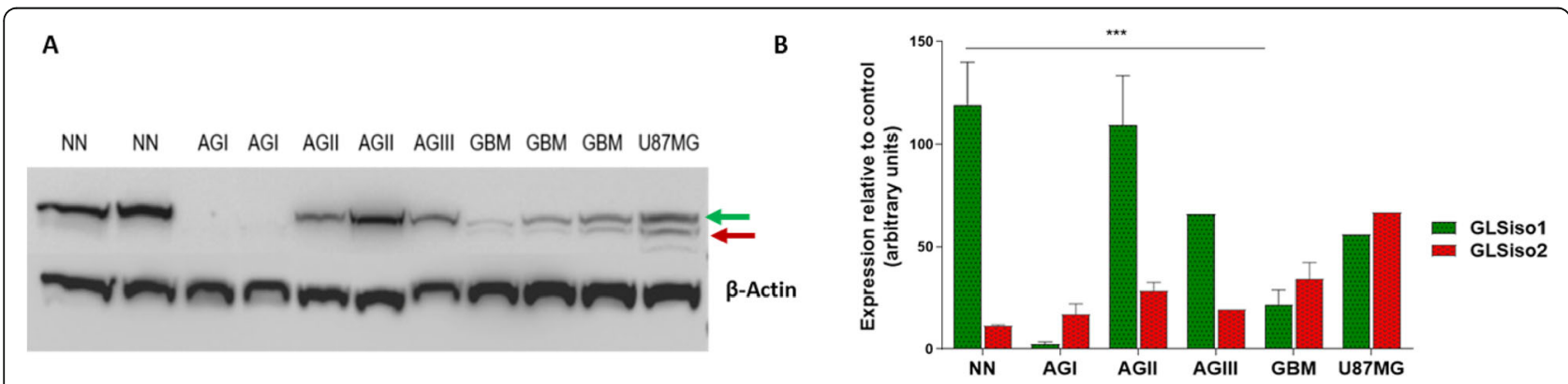

Fig. 3 GLS isoforms expression profile during astrocytoma progression and U87MG human GBM cells. a Western blot analysis of the expression of GLS isoforms in non-neoplastic (NN), pilocytic astrocytoma (AGI), low-grade astrocytoma (AGII), anaplastic astrocytoma (AGIII), glioblastoma (GBM), and U87MG GBM cell line. GLSiso1 and GLSiso2 are indicated by green arrow and red arrows, respectively. $\beta$-actin was used as a loading control. b Quantification of the protein relative to $\beta$-actin protein by ImageJ, represented by mean values \pm standard deviation. The graph is representative of at least two replicates of one experiment. ${ }^{* *} p<0.001$, Two-way ANOVA, Bonferroni post-test 


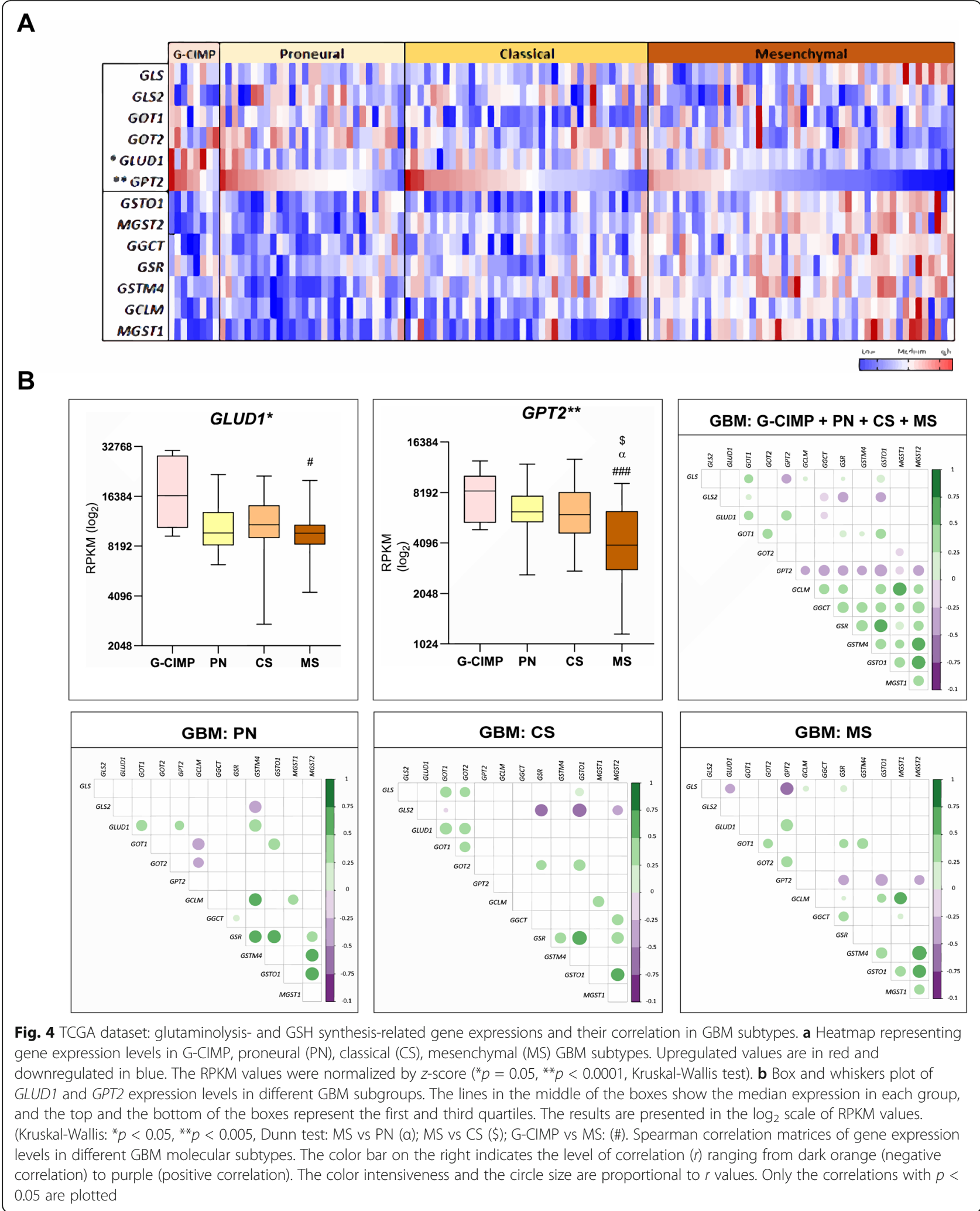


glutathione S-transferase omega 1 (GSTO1), microsomal glutathione S-transferase 1 (MGST1), and microsomal glutathione S-transferase 2 (MSGT2) and analyzed the expression levels in the GBM database of TCGA (Fig. 4a). Additionally, the expression values were correlated to the expression data of glutaminolysis genes (Fig. $4 \mathrm{~b}$ ). The seven genes related to GSH synthesis presented differential expression levels among the GBM molecular subtypes, with statistical differences for all genes $(p<$ 0.0005 , Kruskal-Wallis test). Particularly, the expression of these genes was higher in the MS subtype compared to the other subtypes (Supplemental Figure 2). Moreover, the gene expression levels of GSH synthesis were highly correlated among themselves when all groups of GBM were analyzed together (GBM: G-CIM+PN+CS+ MS), and interestingly, an inverse correlation was noted with GPT2 expression. Particularly in the MS subtype, GPT2 expression correlated inversely to the expression levels of GSTO1, GSR, and MGST2, suggesting the possibility of Glu not converted to $\alpha-K G$ being used for GHS synthesis (Fig. 4b). All statistically significant values are demonstrated on Supplemental Table 2.

\section{GLUD1 upregulation in IDH $1^{\text {mut }}$ AGIII correlated to downregulation of genes involved in GSH synthesis} G-CIMP cases of PN molecular subtype of GBM presented the higher GLUD1 and GPT2 expression levels when compared to the other subgroups. Additionally, genes related to GSH synthesis presented the lowest expression levels in G-CIMP cases. These data and the information that increased conversion of Gln to Glu has been described in glioma cells harboring IDH1 mutation [41] motivated us to investigate the $I D H 1$ mutation status influence in the expression levels of genes involved in the glutaminolysis pathway and GSH. Gene expression levels previously analyzed in GBM cases were also analyzed in AGII and AGIII cases of TCGA, separating cases with and without IDH1 mutation (Fig. 5a). In our cohort, 20 AGII out of 26 cases (77\%) presented IDH1 mutation, and 11 out of 18 AGIII cases harbored IDH1 mutation (61\%). Interestingly, upregulated GLUD1 and GPT2 expressions were observed in $I D H 1^{\text {mut }}$ AGII cases, with a significant difference compared to $I D H 1^{\text {wt }}$ AGII cases for GPT2 expression ( $p<0.05$, Mann-Whitney test), and a trend of increase for GLUD1 (Fig. 5b). In a larger TCGA dataset, with $51 \mathrm{IDH} 1^{\mathrm{mut}}$ AGII out of 63 cases (86\%), and $80 I D H 1^{\text {mut }}$ AGIII out of 129 cases (63\%), a significant higher GLUD1 and GPT2 expression levels were observed both in AGII and AGIII harboring IDH1 mutation when compared to cases without IDH1 mutation $(p<0.01$ and $p<0.0001$ for GLUD1 in AGII and AGIII respectively; $p<0.01$ and $p<0.001$ for GPT2 in AGII and AGIII, respectively, Mann-Whitney test) (Fig. 5b). Expression analyses of expression levels of all genes presented in the correlation matrix demonstrated that $I D H 1^{\text {mut }}$ AGII cases presented activation of both glutaminolysis and GSH synthesis in contrast to $I D H 1^{\text {wt }}$ AGII, with an inverse correlation between GLS2 and MSGT2 expressions in $I D H 1^{\text {mut }}$ AGII (Fig. 5c). On the other hand, $I D H 1^{\text {mut }}$ AGIII cases presented a significantly high correlation between GLUD1 and GPT2 expression levels, and inverse correlations with several genes related to GSH synthesis. Of note, the GLUD1 expression level was inversely correlated to GSR, GCLM, GSTO1, and GSMT2 expression levels, indicating the downregulation of these gene expressions when GLUD1 was upregulated in AGIII cases with IDH1 mutation (Fig. 5c). All statistically significant values are demonstrated on Supplemental Table 3.

GLUD1 and GPT2 protein downregulation in GBM-MS and GLUD1 downregulation in AGII-IDH ${ }^{\text {wt }}$ correlated with upregulation of GS activity

The GBM-MS presented low expression of GLUD1 and GPT2 protein levels when compared to the GBM-PN cases $(p<0.05$ and $p<0.001$, respectively). Although statistical significance was not reached, AGII-IDH ${ }^{\text {wt }}$ presented low GLUD1 protein level (Fig. 6a, b). Additionally, we evaluated whether the level of these proteins correlated with glutathione synthetase (GS) expression. Interestingly, a significant increase of GS expression was observed in GBM-MS and AGII-IDH $H^{\mathrm{wt}}$ in comparison with GBM-PN and AGII-ID $H^{\text {mut }}(p<0.01$ and $p=0.05$, respectively) (Fig. 6c, d). We also found a trend of increased levels of GSH in GBM-MS (IDH $\left.{ }^{\mathrm{wt}}\right)$ samples in contrast to more uniform low GSH levels in GBM-PN $\left(I D H^{\mathrm{mut}}\right)$ (Supplemental Figure 3).

\section{Discussion}

Metabolic reprogramming has been proposed to be a hallmark of cancer [42], and in the present analysis, we observed a progressive activation of the glutaminolysis from low-grade astrocytoma to GBM. Glutaminolysis has been pointed to as one of the major altered metabolic pathways related to tumor growth [12, 16]. High extracellular Gln concentration has been associated with cell transformation [17], and its metabolism was related to cell survival and tumor growth by maintaining redox balance, bioenergetics, and supporting macromolecular biosynthesis $[28,42]$. We have previously reported the upregulation of Gln transporters, ASCT2 and LAT1, in all grades of astrocytoma [20]. Here, we showed the upregulation of the mitochondrial isoform of GLS [39], GLSiso2 (GAC), in all grades of astrocytoma at gene and protein levels, and a gradual increase of its expression was observed in parallel to the increment of malignancy. GLSiso2 involvement in cancer progression has been previously reported in prostate cancer and B cell 


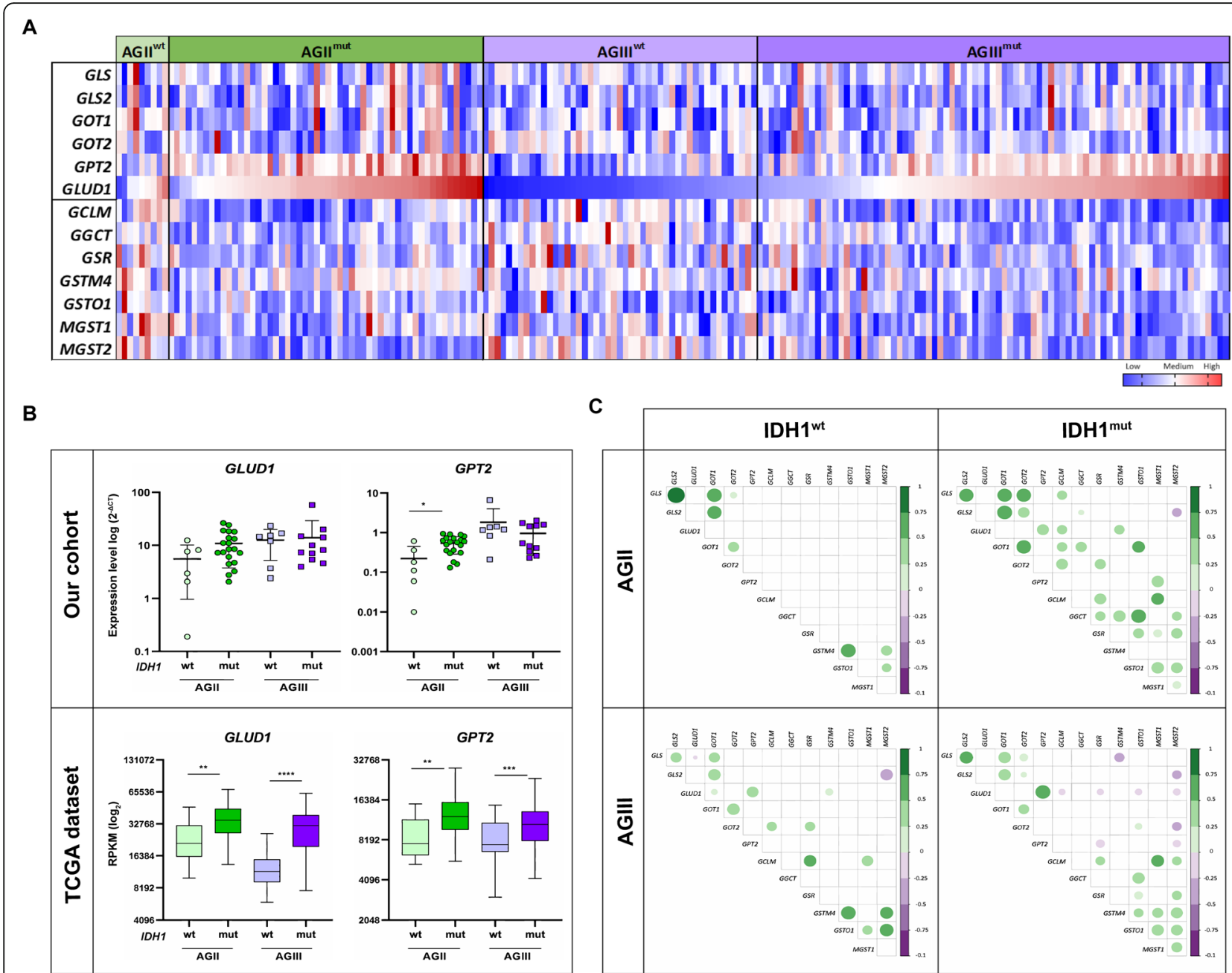

Fig. 5 AGII, AGIII TCGA dataset: glutaminolysis- and GSH synthesis-related gene expressions according to IDHI mutation. a Heatmap representing the expression levels of genes presenting statistical significance in AGII and AGIII with wild type (wt) and mutated IDHI (mut). Upregulated values are represented in red and downregulated in blue. The RPKM values were normalized by $z$-score. $\mathbf{b}$ The differential expression levels of GLUD1 and GPT2 in each stratified group. (Mann-Whitney test: ${ }^{*} p<0.05,{ }^{* *} p<0.01 ;{ }^{* * *} p<0.0005,{ }^{* * *} p<0.0001$ ). Horizontal bars show the median expression in each group for the up panels, while the bottom panel boxes represent the first (top) and third (bottom) quartiles, and the median is represented by the middle line in the boxes. The results are presented in the $\log _{2}$ scale of RPKM values. c Spearman correlation matrix among the gene expression levels of each group. The color bar on the right indicates the level of correlation ranging from dark orange (negative correlation) to blue (positive correlation). The color intensiveness and the circle sizes are proportional to $r$ values. Only the correlations with $p<$ 0.05 are plotted

lymphoma [43]. GLSiso2 is activated by inorganic phosphate [39] and it is also under $\mathrm{c}-M y c$ oncogene influence, through a mechanism involving miRNA [43, 44]. The c-Myc can also upregulate the GLS isoforms KGA and GAC at protein levels increasing the levels of intracellular glutamate in Epstein-Barr virus-infected cells [68]. Oscillation of GLSiso2 expression has been associated with oxygen concentration, with an increase in hypoxic conditions [39]. Our finding of GLSiso2 higher expression in GBM, the more malignant astrocytoma presenting necrosis, corroborated these previous observations. The cytosolic GLSiso1 (KGA) expression was also higher in more malignant than lower-grade astrocytomas. Nevertheless, the high expression observed in NN tissue renders this target less eligible for therapeutic purposes.

The other glutaminase, GLS2, in contrast to GLS with broad distribution among normal tissue, presents a more restricted distribution in the liver, brain, pituitary gland, and pancreas $[45,46]$. The GLS2 expression level was significantly lower in astrocytoma than $\mathrm{NN}$, with the lowest expression in GBM of MS subtype in our cohort. This finding corroborates the tumor suppressor role attributed to GLS2 in previous studies, where inhibition of tumor cell proliferation, colony formation, and migration were attributed to GLS2 [28, 43, 47-51]. This tumor 


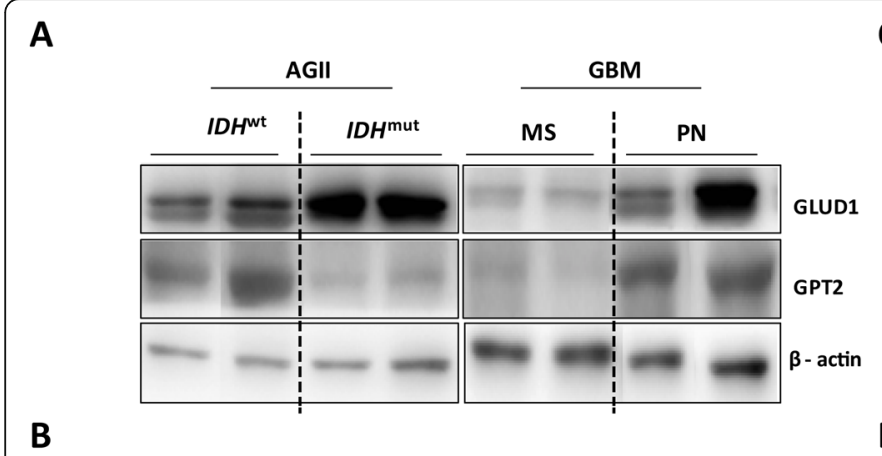

B

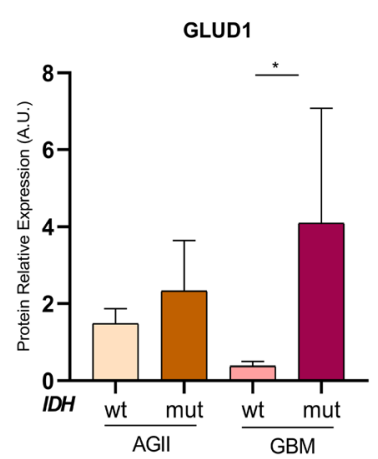

C

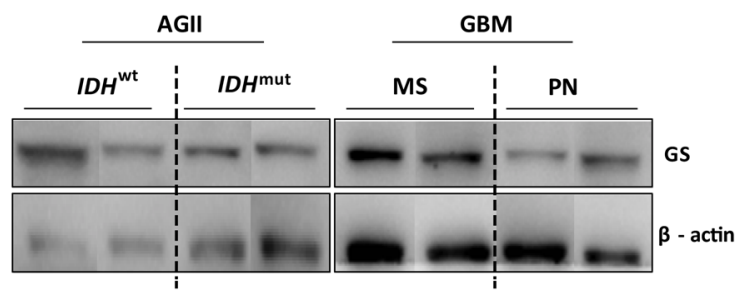

D

Fig. 6 GLUD1, GPT2, and GS protein expression analysis in GBM and low-grade astrocytomas according to IDH mutation status. a Western blotting analysis of the expression of GLUD1 and GPT2 in AGII-IDH $H^{\text {wt }}$, AGII-IDH $H^{\text {mut }}$, GBM-MS (IDH $H^{\text {wt }}$ ) and GBM-PN (IDH ${ }^{\text {mut }}$ ) samples, and GS in c. $\beta$ actin was used as the loading control. $\mathbf{b}$, $\mathbf{d}$ Quantification of each protein relative to $\beta$-actin protein by ImageJ, represented by mean values \pm standard deviation. The graph is representative of at least four replicates of one experiment. ${ }^{*} p<0.05,{ }^{* *} p<0.001$, one-way ANOVA,

Bonferroni post-test

suppressor activity is dependent on p53 and other related proteins, as p63 and p73 [48]. Therefore, concerning the first step of the glutaminolysis pathway, our findings suggested that GLSiso2 plays a key role in tumorigenesis and malignant progression of astrocytoma, whereas the GLS2 expression pattern is consistent with tumor suppressor function, being mostly suppressed in the aggressive MS molecular subtype of GBM.

Interestingly, the downflow activation of the glutaminolysis pathway with the conversion of Glu to $\alpha-K G$ through dehydrogenase and transaminase varied according to the astrocytoma grade. Significant downregulation of GLUD1 and GPT2 expressions were observed in GBM compared to lower-grade astrocytoma in our cohort and confirmed in the TCGA dataset. Particularly, GPT2 was significantly downregulated in GBM of MS subtype compared to other molecular subtypes. We also observed this downregulation of GLUD1 and GPT2 at the protein level in GBM-MS subtype. The downregulation of these proteins may increase intracellular Glu availability, which may be directed for GSH synthesis [52].

GSH is a tripeptide formed by glutamic acid, cysteine, and glycine and plays an important role in the maintenance of the intracellular redox balance [53, 54]. Elevated
GSH levels confer resistance to chemotherapy in various types of cancer [55-57] by binding to or reacting with drugs, interacting with ROS, preventing damage to proteins or DNA, and participating in DNA repair processes [55]. Moreover, GSH- and GSH-related enzymes including synthetase (GS), ligase (GCLM), transferase (GGT), reductase (GSR), and glutathione S-transferases (GSTM4, GSTO1, MGST1, MGST2) activities may play a role in adaptive detoxification processes in response to the oxidative stress, thus contributing to drug resistance phenotype [53, 54].

The increase of intracellular Glu level may favor its release to the extracellular space by a Gln/cysteine antiporter system x c-dependent, which increases intracellular cysteine levels ([Cys]i). In turn, high [Cys]i favors GSH synthesis [52]. The TCGA data analysis showed a significant inverse correlation among GPT2 expression and expression level of several genes related to GSH synthesis. Particularly, upregulation of GSTO1, MGST2, and GSR were correlated significantly to the downregulation of GPT2 in the MS subtype of GBM. Also, we observed a significant increase of the GS protein and a trend of an increase of GSH protein level in the GBM-MS in comparison with the GBM-PN samples. The antioxidative effect provided by increased synthesis of GSH can balance the elevated generation of ROS due to the high metabolic 
rate presented by GBM cells and favor their survival [58]. Such mechanism may be related to the aggressive behavior of GBM of MS molecular subtype.

In contrast, AGII and AGIII presented higher GLUD1 expression levels than GBM, and particularly in those cases harboring $I D H 1$ mutation, and a similar trend was observed at the protein level, although this observation needs to be extended for additional cases to reach statistical significance. Similarly, GPT2 expression was also higher in AGII and AGIII cases harboring IDH1 mutation, but this finding was not confirmed at the protein level in our studied cohort. However, GS protein level was significantly lower in AGII-IDH ${ }^{\text {mut }}$ compared to AGII-IDH $^{\text {wt }}$, suggesting that low-grade astrocytomas harboring IDH mutation may be more susceptible to ROS induced stress. Metabolomic studies of IDH1 mutant cells have revealed alterations in Gln, fatty acid, and citrate synthesis pathways $[59,60]$. IDH1 mutation was

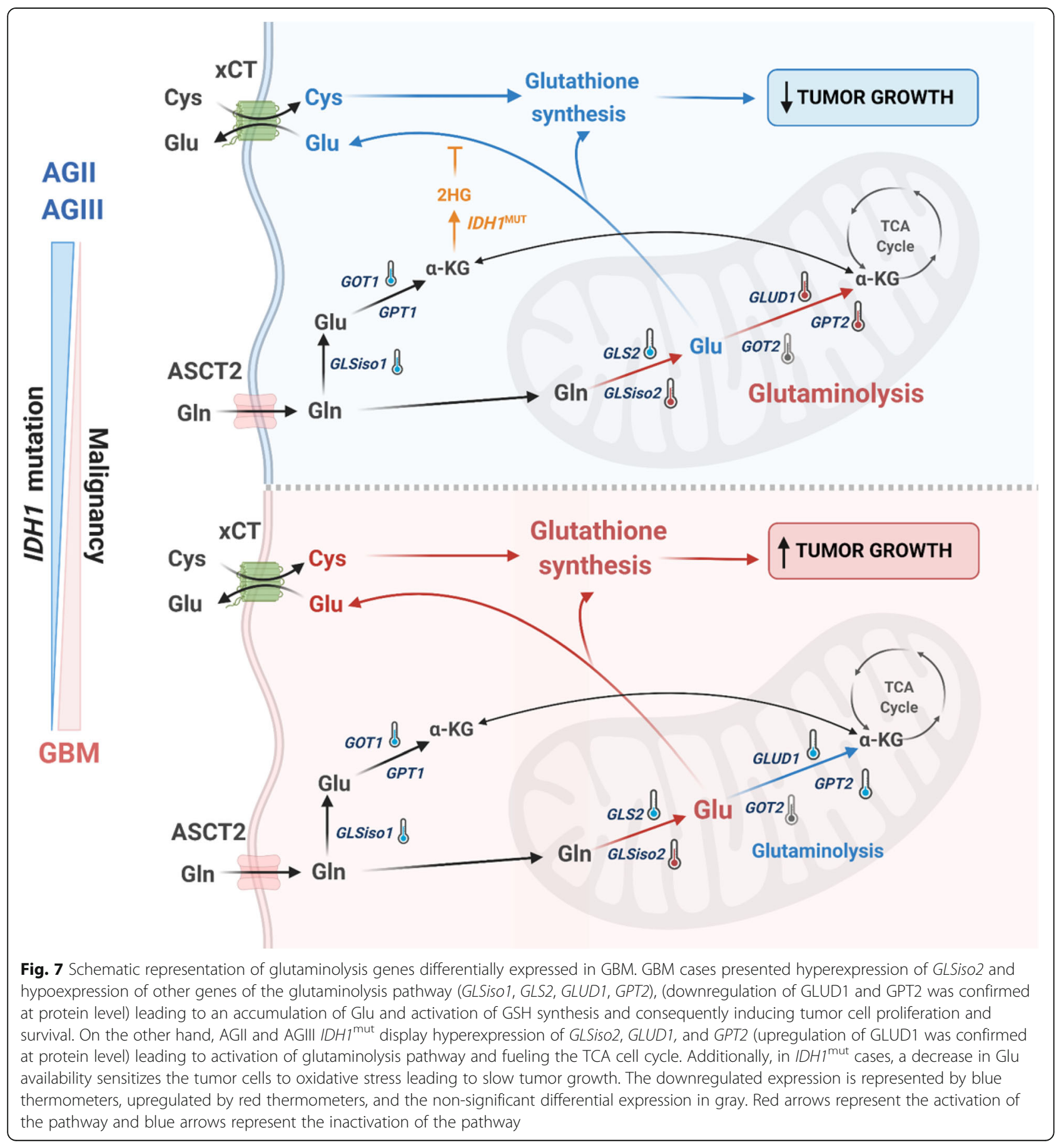


shown to convert $\alpha-K G$ to $D-2-$ hydroxyglutarate, which due to its structural similarities acts as a competitive inhibitor reducing the activity of $\alpha-K G$-processing enzymes [61]. As feedback, $\alpha-K G$ is replenished by glutaminolysis and TCA cycle, which leads to a decrease in Gln and Glu levels [62]. Therefore, $I D H^{\text {mut }}$ gliomas are "glutamate addicted", and the lack of Glu decreases its exchange with Cys through the system $\mathrm{X}_{\mathrm{C}}{ }^{-}$[63]. The lack of cytoplasmic Cys reduces GSH synthesis, which increases the susceptibility to ROS-induced stress as through radiation therapy or TMZ treatment [63]. In this context, reduced Glu contributes to a better outcome presented by gliomas with IDH1 mutation [41, 52]. Our correlation analysis among genes related to glutaminolysis and GSH synthesis-related genes demonstrated that GLUD1 and GPT2 expression levels inversely correlated to GSH synthesis-related gene expression levels, particularly in $I D H 1^{\text {mut }}$ AGIII. Our findings reinforce the hypothesis that decreasing Glu may sensitize $I D H 1^{\text {mut }}$ cells to radiation and ROS-inducing drugs due to reduced GSH synthesis. Indeed, GLS inhibition and $I D H 1$ mutation were recently demonstrated to present a synthetic lethal relationship under conditions of oxidative stress [41].

Our findings together with TCGA data analysis indicated that AGII and AGIII harboring IDH1 mutation may decrease tumor cell fitness by lowering Glu, GSH, and resistance to oxidative stress. Interestingly, the end metabolites of these enzymes, ammonia, and alanine are measurable by the MR spectroscopy [64-67]. Thus, monitoring the waning of GLUD1 and GPT2 expression levels by measuring their end substrates by this noninvasive imaging technique may potentially detect the progression of lower-grade astrocytomas harboring IDH1 mutation towards secondary GBM, and it would, therefore, allow a change in the therapeutic strategy for these patients. Such hypothesis would be worthwhile to test in future studies.

\section{Conclusion}

In conclusion, GLSiso2 upregulation was associated with tumorigenesis and tumor progression in astrocytomas. Particularly in GBM, the accumulation of Glu due to GPT2 and GLUD1 downregulation correlated to upregulation of genes related to GSH synthesis which could favor tumor cell survival, mostly in the most aggressive MS subtype. In contrast, GLUD1 may lead to a decrease in GSH synthesis in $I D H 1^{\text {mut }}$ low-grade astrocytomas increasing the susceptibility to oxidative stress, rendering them more sensitive to radiation therapy and to alkylating therapy (Fig. 7).

\section{Abbreviations}

GBM: Glioblastoma; AGI: Astrocytoma grade I; AGIl: Astrocytoma grade II; AGIII: Astrocytoma grade III; NN: Non-neoplastic; Gln: Glutamine;
Glu: Glutamate; TCA: Tricarboxylic acid; a-KG: Alpha ketoglutarate; GSH: Glutathione; GLS: Glutaminase; GLS2: Glutaminase 2;

GLSiso1: Glutaminase isoform 1; GLSiso2: Glutaminase isoform 2; GLUD1: Glutamate dehydrogenase 1; GOT1: Glutamate oxaloacetate transaminase 1; GOT2: Glutamate oxaloacetate transaminase 2; GPT1: Glutamate pyruvate transaminase 1; GPT2: Glutamate pyruvate transaminase 2; TCGA: The Cancer Genome Atlas; IDH: Isocitrate dehydrogenase; IDH1: Isocitrate dehydrogenase 1; IDH1 ${ }^{\text {MUT: }}$ : Isocitrate dehydrogenase 1 mutation; IDH ${ }^{\mathrm{WT}}$ : Isocitrate dehydrogenase wild type; CNS: Central nervous system; WHO: World Health Organization; NF1: Neurofibromin 1; RB1: Retinoblastoma 1; TMZ: Temozolomide; qRTPCR: Quantitative reverse transcription PCR; ASCT2 (SLC1A5): Solute carrier family 1 member 5; LAT1 (SLC7A5): Solute carrier family 7 member 5; HPRT: Hypoxanthine phosphoribosyltransferase; GUSB: Glucuronidase beta; TBP: TATA box binding protein; PCR: Polymerase chain reaction; ATCC: American Type Culture Collection; mRNA: Messenger RNA; ROC: Receiver operating characteristic; PN: Proneural; CS: Classical; MS: Mesenchymal; G-CIMP: Glioma CpG island methylator phenotype; CGLM: Glutamate-cysteine ligase modifier subunit; GGCT: Gammaglutamylcyclotransferase; GSTM4: Glutathione S-transferase mu 4; GSTO1: Glutathione S-transferase omega 1; MGST1: Microsomal glutathione S-transferase 1; MGST2: Microsomal glutathione S-transferase 2;

GSR: Glutathione-disulfide reductase; GS: Glutathione synthetase; ROS: Reactive oxygen species; MR: Magnetic resonance

\section{Supplementary Information}

The online version contains supplementary material available at https://doi. org/10.1186/s40170-021-00255-8.

Additional file 1: Figure S1. Expression analysis of genes related to glutaminolysis in different molecular subtypes of GBM in our cohort. A: Heatmap of GLSiso 1, GLSiso2, GLS, GLS2, GOT1, GOT2, GPT2, and GLUD1 mRNA expression levels in different molecular subtypes of GBM (PN: proneural, CS: classical, MS: mesenchymal). Upregulated values are in red and downregulated in blue. The RPKM values were normalized by zscore. B: GLS2 expression differed significantly among the subtypes (Kruskal-Wallis, $p<0.005,{ }^{* *} p<0.05$, Dunn test). Horizontal bars show the median relative expression in each group.

Additional file 2: Figure S2. Expression analysis of GPT2 and genes related to glutathione synthesis in different molecular subgroups of GBM from TCGA RNAseq dataset. Box and whiskers plot of GPT2, GCLM, GGCT, GSR, GSTM4, GSTO1, MGST1, and MGST2 expression levels in G-CIMP, proneural (PN), classical (CS), and mesenchymal (MS) GBM cases. The top and the bottom of boxes represent the first and third quartiles, respectively, and the lines in the middle the median of the groups. KruskalWallis: ${ }^{*} p<0.05,{ }^{* *} p<0.005,{ }^{* *} p<0.0005$, Dunn test: CS vs PN (\#); MS vs PN (1); MS vs CS (\$); G-CIMP vs MS: ( $\neq)$; G-CIMP vs CS: (@). The results are presented in the log2 scale of RPKM values.

Additional file 3: Figure S3 Analysis of Reduced Glutathione (GSH) levels in $A G I I D H^{w t}, A G I I D H^{\text {mut }}, G B M-M S\left(I D H^{w t}\right)$ and PN (IDH $\left.{ }^{\text {mut }}\right)$. GSH levels [pmol/Mg of total protein] analysis of low-grade astrocytoma IDH ${ }^{\text {wt }}$, low grade astrocytoma IDH ${ }^{\text {mut }}$, Glioblastoma ${ }^{\text {wt }}$-Mesenchymal, and glioblastoma IDH ${ }^{\text {mut }}$-Proneural). Results are presented as mean \pm standard deviation ( $n \geq 3$ samples of each group).

Additional file 4: Supplemental Table S1. Correlations values of the glutaminolysis pathway genes in all grades of astrocytoma.

Additional file 5: Supplemental Table S2. Correlations values of the glutaminolysis and GSH pathway genes in the subtypes of GBM.

Additional file 6: Supplemental Table S3. Correlations values of the glutaminolysis and GSH pathway genes in AGII and AGIII with IDH1 wild type $\left(I D H T^{w t}\right)$ and mutated IDHI (IDHI $\left.{ }^{\text {mut }}\right)$.

Acknowledgements

We thank the doctors and residents from the Discipline of Neurosurgery of the Department of Neurology at Hospital das Clinicas of School of Medicine, the University of São Paulo, for their help in tumor sample collection. 


\section{Authors' contributions}

Conception, design, and writing: YEMF and SKNM. Development of methodology: MJA, MU, AFS, MSB, SMOS, and SKNM. Acquisition of data: MJA, MU, AFS, and GSA. Analysis and interpretation of data (i.e.. computational analysis, statistics): AML, SS, IFM, YEMF, MSB, and SKNM. Revision of the manuscript: YEMF, MTL, SMOS, and SKNM. The authors read and approved the final manuscript.

\section{Funding}

We are grateful to the São Paulo Research Foundation (FAPESP, grant processes n²001/12898-4, 2004/12133-6, 2013/02162-8 (SKNM), 2014/501375 (SKNM), 2013/07937-8 (MSB), 2020/02988-7 (SKNM), 2015/26328-8 (MTL)); to the Conselho Nacional de Desenvolvimento Científico e Tecnológico (CNPq, grant number 14/2011483467/2011-1, 305730/2015-0, 870255/1997-5); to the Coordenação de Aperfeiçoamento de Pessoal de Nível Superior (CAPES, grant number NUFFIC 062/15 (SKNM), Processes no 9999.001625/2015-02 (SKNM), 88887.321693/2019-00 (MTL)); and to the Fundação Faculdade de Medicina (FFM-USP)

\section{Availability of data and materials}

Normalized gene expression data are available from the corresponding author on request. The results shown here are in part based upon data generated by the TCGA Research Network: https://www.cancer.gov/tcga.

\section{Declarations}

\section{Ethics approval and consent to participate}

The procedures were performed with informed and approved consents according to the Institutional Ethical Committee guidelines at the Hospital das Clinicas of the School of Medicine of University of São Paulo (691/05).

\section{Consent for publication}

Not applicable

\section{Competing interests}

The authors declare that they have no competing interests.

\section{Author details}

'Laboratory of Molecular and Cellular Biology (LIM 15), Department of Neurology, Faculdade de Medicina FMUSP, Universidade de São Paulo, São Paulo 01246-903, Brazil. 'Department of Molecular Pharmacology, University of Groningen, 9713 Av, Groningen, The Netherlands. ${ }^{3}$ School of Applied Mathematics, Fundação Getulio Vargas, Rio de Janeiro 22250-900, Brazil. ${ }^{4}$ Institute of Chemistry, Department of Biochemistry, Universidade de São Paulo, CEP, São Paulo 05508-000, Brazil. ${ }^{5}$ Department of Internal Medicine, Division of Metabolism, Endocrinology, and Diabetes, University of Michigan, Ann Arbor, Michigan, USA.

\section{Received: 27 June 2020 Accepted: 1 April 2021}

\section{Published online: 28 April 2021}

\section{References}

1. Siegel RL, Miller KD, Jemal A. Cancer statistics, 2019. CA Cancer J Clin. 2019; 69(1):7-34.

2. Dolecek TA, Propp JM, Stroup NE, Kruchko C. CBTRUS Statistical report: primary brain and central nervous system tumors diagnosed in the United States in 2005-2009. Neuro Oncol. 2012;14(suppl 5):v1-49.

3. Miranda-Filho A, Piñeros M, Soerjomataram I, Deltour I, Bray F. Cancers of the brain and CNS: global patterns and trends in incidence. Neuro Oncol. 2016;19(2):now166.

4. Jones C, Perryman L, Hargrave D. Paediatric and adult malignant glioma: close relatives or distant cousins? Nat Rev Clin Oncol. 2012;9(7):400-13.

5. Molinaro AM, Taylor JW, Wiencke JK, Wrensch MR. Genetic and molecular epidemiology of adult diffuse glioma. Nat Rev Neurol. 2019;15(7):405-17.

6. Sanai N, Alvarez-Buylla A, Berger MS. Neural stem cells and the origin of gliomas. N Engl J Med. 2005;353(8):811-22.

7. Wang Q, Hu B, Hu X, Kim H, Squatrito M, Scarpace L, et al. Tumor evolution of glioma-intrinsic gene expression subtypes associates with immunological changes in the microenvironment. Cancer Cell. 2017;32(1):42-56 e6.

8. Verhaak RGW, Hoadley KA, Purdom E, Wang V, Qi Y, Wilkerson MD, et al. Integrated Genomic Analysis Identifies Clinically Relevant Subtypes of
Glioblastoma Characterized by Abnormalities in PDGFRA, IDH1, EGFR, and NF1. Cancer Cell. 2010;17(1):98-110.

9. Yan H, Parsons DW, Jin G, McLendon R, Rasheed BA, Yuan W, et al. IDH1 and IDH2 mutations in gliomas. N Engl J Med. 2009;360(8):765-73.

10. Sidaway P. Glioblastoma subtypes revisited. Nat Rev Clin Oncol. 2017;14(10): 587-7.

11. Touat M, Li YY, Boynton AN, Spurr LF, lorgulescu JB, Bohrson CL, et al. Mechanisms and therapeutic implications of hypermutation in gliomas. Nature. 2020;580(7804):517-23.

12. Strickland M, Stoll EA. Metabolic Reprogramming in Glioma. Front Cell Dev Biol. 2017;5.

13. Vander Heiden M, Cantley L, Thompson C. Understanding the Warburg effect: The metabolic Requiremetns of cell proliferation. Science (80). 2009: 324(5930):1029-33.

14. Oruganty K, Campit SE, Mamde S, Lyssiotis CA, Chandrasekaran S. Common biochemical properties of metabolic genes recurrently dysregulated in tumors. Cancer Metab. 2020;8(1):1-15.

15. Altman BJ, Stine ZE, Dang CV. From Krebs to clinic: glutamine metabolism to cancer therapy. Nat Publ Gr. 2016;16(10):619-34.

16. Cluntun AA, Lukey MJ, Cerione RA, Locasale JW. Glutamine metabolism in cancer: understanding the heterogeneity. Trends in Cancer. 2017;3(3): $169-80$.

17. Jin L, Kang S. Glutaminolysis as a target for cancer therapy. Oncogene. 2016; 35(28):3619-25.

18. DeBerardinis RJ, Sayed N, Ditsworth D, Thompson CB. Brick by brick: metabolism and tumor cell growth. Curr Opin Genet Dev. 2008;18(1):54-61.

19. Herranz D. Glutaminolysis gets the spotlight in cancer. 2017:8(7):10761-2.

20. Alves MJF, Uno M, da Silva R, Oba-Shinjo SM, Marie SKN. The expression of the aminoacid transporters ASCT2 (SLC1A5) and LAT1 (SLC7A5) in astrocytomas. Med Express. 2016;3(6):1-8.

21. Vander Heiden MG. Targeting cancer metabolism: a therapeutic window opens. Nat Rev Drug Discov. 2011;10(9):671-84.

22. Daye D, Wellen KE. Metabolic reprogramming in cancer: unraveling the role of glutamine in tumorigenesis. Semin Cell Dev Biol. 2012;23(4):362-9.

23. Ralph J. DeBerardinis, and TC. Q's next: The diverse functions of glutamine in metabolism, cell biology and cancer. 2010;29(3):313-324.

24. Luengo A, Gui DY, Vander Heiden MG. Targeting metabolism for cancer therapy. Cell Chem Biol. 2017:24(9):1161-80

25. Gross Ml, Demo SD, Dennison JB, Chen L, Chernov-Rogan T, Goyal B, et al. Antitumor activity of the glutaminase inhibitor CB-839 in triple-negative breast cancer. Mol Cancer Ther. 2014;13(4):890-901.

26. Jacque N, Ronchetti AM, Larrue C, Meunier G, Birsen R, Willems L, et al. Targeting glutaminolysis has antileukemic activity in acute myeloid leukemia and synergizes with BCL-2 inhibition. Blood. 2015;126(11): $1346-56$.

27. Xiang Y, Stine ZE, Xia J, Lu Y, O'Connor RS, Altman BJ, et al. Targeted inhibition of tumor-specific glutaminase diminishes cell-autonomous tumorigenesis. J Clin Invest. 2015;125(6):2293-306.

28. Obara-Michlewska M, Szeliga M. Targeting glutamine addiction in Gliomas. Cancers (Basel). 2020;12:2.

29. Walker MC, van der Donk WA. The many roles of glutamate in metabolism. J Ind Microbiol Biotechnol. 2016;43(2-3):419-30.

30. Lv H, Zhen C, Liu J, Yang P, Hu L, Shang P. Unraveling the potential role of glutathione in multiple forms of cell death in cancer therapy. Oxid Med Cell Longev. 2019;2019:1-16.

31. Wang Z, Ding Y, Wang X, Lu S, Wang C, He C, et al. Pseudolaric acid B triggers ferroptosis in glioma cells via activation of Nox4 and inhibition of xCT. Cancer Lett. 2018;428:21-33.

32. Lewerenz J, Hewett SJ, Huang Y, Lambros M, Gout PW, Kalivas PW, et al. The cystine/glutamate antiporter system $\times \mathrm{c}-$ in health and disease: from molecular mechanisms to novel therapeutic opportunities. Antioxid Redox Signal. 2013;18(5):522-55.

33. Louis DN, Ohgaki H, Wiestler OD, Cavenee WK, Burger PC, Jouvet A, et al. The 2007 WHO classification of tumours of the central nervous system. Acta Neuropathol. 2007;114(2):97-109.

34. Valente V, Teixeira SA, Neder L, Okamoto OK, Oba-Shinjo SM, Marie SK, et al. Selection of suitable housekeeping genes for expression analysis in glioblastoma using quantitative RT-PCR. BMC Mol Biol. 2009;10(1):17.

35. Livak KJ, Schmittgen TD. Analysis of relative gene expression data using real-time quantitative $P C R$ and the $2-\Delta \Delta C T$ method. Methods. 2001;25(4): $402-8$. 
36. Matés JM, Segura JA, Campos-Sandoval JA, Lobo C, Alonso L, Alonso FJ, et al. Glutamine homeostasis and mitochondrial dynamics. Int J Biochem Cell Biol. 2009;41(10):2051-61.

37. Katt WP, Cerione RA. Glutaminase regulation in cancer cells: A druggable chain of events. Drug Discov Today. 2014;19(4):450-7.

38. Vijayakumar SN, Sethuraman S, Krishnan UM. Metabolic pathways in cancers: key targets and implications in cancer therapy. RSC Adv. 2015;5(52):4175162.

39. Cassago A, Ferreira APS, Ferreira IM, Fornezari C, Gomes ERM, Greene KS, et al. Mitochondrial localization and structure-based phosphate activation mechanism of Glutaminase C with implications for cancer metabolism. Proc Natl Acad Sci. 2012;109(4):1092-7.

40. Galatro TF, et al. Correlation between molecular features and genetic subtypes of Glioblastoma: critical analysis in 109 cases. Med. Express. 2017;4.

41. McBrayer SK, Mayers JR, DiNatale GJ, Shi DD, Khanal J, Chakraborty AA, et al. Transaminase inhibition by 2-hydroxyglutarate impairs glutamate biosynthesis and redox homeostasis in glioma. Cell. 2018;175(1):101-16 e25.

42. Pavlova NN, Thompson CB. The emerging hallmarks of cancer metabolism. Cell Metab. 2016:23(1):27-47.

43. Gao P, Tchernyshyov I, Chang T-C, Lee Y-S, Kita K, Ochi T, et al. c-Myc suppression of miR-23a/b enhances mitochondrial glutaminase expression and glutamine metabolism. Nature. 2009 Apr 15;458(7239):762-5.

44. Márquez J, Alonso FJ, Matés JM, Segura JA, Martín-Rufián M, CamposSandoval JA. Glutamine Addiction In Gliomas. Neurochem Res. 2017;42(6): $1735-46$.

45. Curthoys NP. Regulation of glutaminase activity and glutamine metabolism. Annu Rev Nutr. 1995;15(1):133-59.

46. Ardlie KG, Deluca DS, Segre AV, Sullivan TJ, Young TR, Gelfand ET, et al. The genotype-tissue expression (GTEx) pilot analysis: multitissue gene regulation in humans. Science. 2015;348(6235):648-60.

47. Suzuki S, Tanaka T, Poyurovsky MV, Nagano H, Mayama T, Ohkubo S, et al. Phosphate-activated glutaminase (GLS2), a p53-inducible regulator of glutamine metabolism and reactive oxygen species. Proc Natl Acad Sci. 2010;107(16):7461-6

48. Hu W, Zhang C, Wu R, Sun Y, Levine A, Feng Z. Glutaminase 2, a novel p53 target gene regulating energy metabolism and antioxidant function. Proc Natl Acad Sci. 2010;107(16):7455-60.

49. Szeliga M, Bogacińska-Karaś M, Kuźmicz K, Rola R, Albrecht J. Downregulation of GLS2 in glioblastoma cells is related to DNA hypermethylation but not to the p53 status. Mol Carcinog. 2016;55(9):1309-16.

50. Xiao D, Ren P, Su H, Yue M, Xiu R, Hu Y, et al. Myc promotes glutaminolysis in human neuroblastoma through direct activation of glutaminase 2. Oncotarget. 2015;6:38.

51. Wang J-B, Erickson JW, Fuji R, Ramachandran S, Gao P, Dinavahi R, et al. Targeting mitochondrial glutaminase activity inhibits oncogenic transformation. Cancer Cell. 2010;18(3):207-19.

52. Maus A, Peters GJ. Glutamate and a-ketoglutarate: key players in glioma metabolism. Amino Acids. 2017:49(1):21-32.

53. Forman $\mathrm{HJ}$, Zhang $\mathrm{H}$, Rinna A. Glutathione: Overview of its protective roles, measurement, and biosynthesis. Mol Aspects Med. 2009;30(1-2):1-12.

54. Meister A. Glutathione metabolism. In 1995. p. 3-7.

55. Traverso N, Ricciarelli R, Nitti M, Marengo B, Furfaro AL, Pronzato MA, et al. Role of glutathione in cancer progression and chemoresistance. Oxid Med Cell Longev. 2013.

56. Rocha CRR, Garcia CCM, Vieira DB, Quinet A, de Andrade-Lima LC, Munford $\checkmark$, et al. Glutathione depletion sensitizes cisplatin- and temozolomideresistant glioma cells in vitro and in vivo. Cell Death Dis. 2014;5(10):e1505-5.

57. Estrela JM, Ortega A, Obrador E. Glutathione in cancer biology and therapy. Crit Rev Clin Lab Sci. 2006:43(2):143-81.

58. Nguyen T-L, Durán R V. Glutamine metabolism in cancer therapy. Cancer Drug Resist. 2018;

59. Borodovsky A, Seltzer MJ, Riggins GJ. Altered cancer cell metabolism in gliomas with mutant IDH1 or IDH2. Curr Opin Oncol. 2012;24(1):83-9.

60. Garrett M, Sperry J, Braas D, Yan W, Le TM, Mottahedeh J, et al. Metabolic characterization of isocitrate dehydrogenase (IDH) mutant and IDH wildtype gliomaspheres uncovers cell type-specific vulnerabilities. Cancer Metab. 2018;6(1):4.

61. Peeters TH, Lenting $K$, Breukels V, van Lith SAM, van den Heuvel CNAM, Molenaar $\mathrm{R}$, et al. Isocitrate dehydrogenase 1-mutated cancers are sensitive to the green tea polyphenol epigallocatechin-3-gallate. Cancer Metab. 2019; $7(1): 4$.
62. Ohka F, Ito M, Ranjit M, Senga T, Motomura A, Motomura K, et al. Quantitative metabolome analysis profiles activation of glutaminolysis in glioma with IDH1 mutation. Tumor Biol. 2014;35(6):5911-20.

63. van Lith SAM, Navis AC, Verrijp $K$, Niclou SP, Bjerkvig R, Wesseling $P$, et al, Glutamate as chemotactic fuel for diffuse glioma cells: Are they glutamate suckers? Biochim Biophys Acta - Rev Cancer. 2014;1846(1):66-74.

64. Albers MJ, Bok R, Chen AP, Cunningham CH, Zierhut ML, Zhang W, et al. Hyperpolarized 13C lactate, pyruvate, and alanine: noninvasive biomarkers for prostate cancer detection and grading. Cancer Res. 2008;68(20):8607-15.

65. Brindle KM, Bohndiek SE, Gallagher FA, Kettunen MI. Tumor imaging using hyperpolarized 13C magnetic resonance spectroscopy. Magn Reson Med. 2011;66(2):505-19.

66. Crisi G. 1 H MR Spectroscopy of meningiomas at 3.0T: the role of glutamate-glutamine complex and glutathione. Neuroradiol J. 2011;24(6): 846-53.

67. Hazany S, Hesselink JR, Healy JF, Imbesi SG. Utilization of glutamate/creatine ratios for proton spectroscopic diagnosis of meningiomas. Neuroradiology. 2007:49(2):121-7.

68. Krishna G, Soman Pillai V, Valiya Veettil M. Upregulation of GLS1 isoforms KGA and GAC facilitates mitochondrial metabolism and cell proliferation in Epstein-Barr virus infected cells. Viruses. 2020;12:811.

\section{Publisher's Note}

Springer Nature remains neutral with regard to jurisdictional claims in published maps and institutional affiliations.
Ready to submit your research? Choose BMC and benefit from:

- fast, convenient online submission

- thorough peer review by experienced researchers in your field

- rapid publication on acceptance

- support for research data, including large and complex data types

- gold Open Access which fosters wider collaboration and increased citations

- maximum visibility for your research: over $100 \mathrm{M}$ website views per year

At $\mathrm{BMC}$, research is always in progress.

Learn more biomedcentral.com/submissions 